\title{
A classificação de Risco segundo a percepção do usuário dos serviços de saúde de uma Unidade de Pronto atendimento em Imperatriz - MA, Brasil
}

Risk classification according to the user's perception of health services in an early care unit in Imperatriz - MA, Brazil

Clasificación de riesgo según la percepción del usuario de los servicios de salud en una unidad de atención temprana de Imperatriz - MA, Brasil

\author{
Wallace Ferreira da Silva \\ ORCID: https://orcid.org/0000-0001-5253-6542 \\ Universidade Ceuma, Brasil \\ E-mail: wallace.ferreiras@hotmail.com \\ Sâmia Marques Lopes \\ ORCID: https://orcid.org/0000-0002-3430-5350 \\ Universidade da Amazônia, Brasil \\ E-mail: samiatocantins@gmail.com \\ Marileide Maciel Correa \\ ORCID: https://orcid.org/0000-0002-2169-8204 \\ Universidade do Sul do Maranhão, Brasil \\ E-mail: edielirammpc@gmail.com \\ Francisco Alves Lima Junior \\ ORCID: https://orcid.org/0000-0002-3117-4949 \\ Universidade Ceuma, Brasil \\ E-mail: Francisco.enfdotrabalho@gmail.com \\ Cristina Limeira Leite \\ ORCID: https://orcid.org/0000-0002-7321-1496 \\ Universidade Ceuma, Brasil \\ E-mail: crislimeira@gmail.com
}

\begin{abstract}
Resumo
A importância dessa pesquisa justifica-se por tratar de um tema de grande relevância para o aprofundamento do estudo relacionado ao tema. Os objetivos geral desse trabalho é ressaltar a importância da classificação de risco, identificando o conhecimento dos usuários acerca da mesma, visando evitar danos à saúde dos pacientes decorrentes da insatisfação ocasionada muitas vezes pela classificação dos usuários recebida durante a triagem, em uma Unidade de Pronto Atendimento UPA localizada no município de Imperatriz-MA; e os específicos: Caracterizar o que é classificação de risco em saúde e quais os riscos avaliados; Conhecer a percepção dos usuários em relação à finalidade da classificação de risco em saúde; Destacar a importância da triagem nos atendimentos em saúde e com isso melhorar os desfechos classificação de risco. A metodologia do presente trabalho consiste em uma abordagem quantitativa. Como procedimento metodológico, a pesquisa será do tipo descritiva e quanto aos procedimentos técnicos será do tipo pesquisa tipo survey. E exploratória pois será também embasada na pesquisa bibliográfica, utilizando-se de material científico, doutrina, revistas e sites da web. Os resultados neste trabalho, foi privilegiada as literaturas que vão ao encontro da classificação de risco, do qual é uma metodologia de trabalho ligada a área de classificação de risco e que a peça-chave de atendimento é o enfermeiro, profissional de nível superior que ordena, classifica, faz o diagnóstico de casos que adentram a um determinado pronto socorro ou até mesmo uma Unidade de Pronto Atendimento (UPA). Onde se chegou à conclusão dos resultados da pesquisa e assim, dos 100 participantes que responderam ao questionário a respeito se conheciam a importância da Triagem para o atendimento, 68\% responderam que sim, e $32 \%$ que não sabiam da importância da Triagem para o atendimento. No que se refere a classificação de risco, $52 \%$ responderam que sim enquanto $48 \%$ afirmaram não saberem para que serve a classificação de risco. Assim, a partir dos discursos elaborados pelos sujeitos, foi possível perceber a percepção deles quanto ao atendimento na UPA São José conforme os gráficos mostram.
\end{abstract}

Palavras-chave: Classificação de risco; Usuários; UPA.

\footnotetext{
Abstract

The importance of this research is justified because it deals with a topic of great relevance for furthering the study related to the topic. The general objectives of this work are to emphasize the importance of risk classification, identifying users' knowledge about it, in order to prevent damage to the health of patients resulting from the
} 
dissatisfaction often caused by the classification of users received during screening, in an Emergency Unit UPA service located in the municipality of Imperatriz-MA; and the specific ones: Characterize what is a health risk classification and what are the assessed risks; Knowing the users' perception regarding the purpose of the health risk classification; Highlight the importance of screening in health care and thus improve risk classification outcomes. The methodology of this work consists of a quantitative approach. As a methodological procedure, the research will be of the descriptive type and regarding the technical procedures, it will be of the survey type research. It is exploratory as it will also be based on bibliographic research, using scientific material, doctrine, journals and websites. The results of this work were privileged the literatures that meet the risk classification, which is a work methodology linked to the area of risk classification and that the key part of care is the nurse, a higher-level professional who orders , classifies, diagnoses cases that enter a particular emergency room or even an Emergency Care Unit (UPA). Where the conclusion of the survey results was reached and thus, of the 100 participants who answered the questionnaire about whether they knew the importance of screening for care, $68 \%$ answered yes, and $32 \%$ did not know the importance of screening for care. With regard to risk classification, 52\% answered yes, while $48 \%$ said they did not know what the risk classification was for. Thus, from the speeches elaborated by the subjects, it was possible to perceive their perception regarding the service at the UPA São José, as shown in the graphs.

Keywords: Risk classification; Users; UPA.

\section{Resumen}

La importancia de esta investigación se justifica porque se trata de un tema de gran relevancia para avanzar en el estudio relacionado con el tema. Los objetivos generales de este trabajo son enfatizar la importancia de la clasificación de riesgos, identificando el conocimiento de los usuarios sobre el mismo, con el fin de evitar daños a la salud de los pacientes derivados de la insatisfacción que muchas veces genera la clasificación de usuarios recibida durante el cribado, en una Emergencia. Unidad de servicio UPA ubicada en el municipio de Imperatriz-MA; y los específicos: Caracterizar qué es una clasificación de riesgo para la salud y cuáles son los riesgos evaluados; Conocer la percepción de los usuarios sobre el propósito de la clasificación de riesgo para la salud; Resaltar la importancia del cribado en la atención médica y así mejorar los resultados de la clasificación de riesgos. La metodología de este trabajo consiste en un enfoque cuantitativo. Como procedimiento metodológico, la investigación será de tipo descriptivo y en cuanto a los procedimientos técnicos será de tipo encuesta. Es exploratorio ya que también se basará en la investigación bibliográfica, utilizando material científico, doctrina, revistas y sitios web. Los resultados de este trabajo se privilegiaron las literaturas que cumplen con la clasificación de riesgo, que es una metodología de trabajo ligada al área de clasificación de riesgo y que la parte clave del cuidado es la enfermera, un profesional de nivel superior que ordena, clasifica, diagnostica casos que ingresan a una sala de emergencias en particular o incluso a una Unidad de Atención de Emergencias (UPA). Cuando se llegó a la conclusión de los resultados de la encuesta y, por lo tanto, de los 100 participantes que respondieron el cuestionario sobre si conocían la importancia del cribado para la atención, el 68\% respondió que sí y el 32\% no conocía la importancia del cribado para la atención. Con respecto a la clasificación de riesgo, el 52\% respondió que sí, mientras que el 48\% dijo que no sabía para qué era la clasificación de riesgo. Así, a partir de los discursos elaborados por los sujetos, fue posible percibir su percepción sobre el servicio en la UPA São José, como se muestra en los gráficos.

Palabras clave: Clasificación de riesgo; Usuarios; UPA.

\section{Introdução}

A classificação de risco é um protocolo pautado em um dos princípios do SUS, a equidade, possibilitando detectar o nível de gravidade e/ou risco de cada paciente, e assim que eles sejam atendidos conforme a sua necessidade. Dessa forma, é importante que a classificação de risco seja realizada corretamente em toda unidades de saúde onde prestam atendimento aos usuários (dos Santos et al, 2020).

Todos os dias nos hospitais, há demandas que por vezes poderiam ser resolvidos em outros níveis de assistência. Daí se percebe, a falta de conhecimento da população no que tange aos níveis de atenção ofertados em saúde, e onde procurar ajudar de acordo com seu estado de saúde. Ou seja, muitas vezes o cliente busca atendimento numa unidade de saúde de alta complexidade e seu problema poderia ser resolvido ainda atenção primária. O cliente tendo esse conhecimento, irá impactar em menos superlotação nos Hospitais (Melo, 2011).

Conforme (Mackway-Jones et al.,2006) Que trata da classificação de risco segundo Manchester, a triagem é um sistema de gerenciamento de risco clínico, implementado em todo o mundo para gerenciar fluxo de paciente com segurança, quando a necessidade exceder a capacidade. Atualmente existe quatro sistemas de classificação de risco, a saber: Emergency Severity Index-ESI (utilizado nos Estados Unidos desde 1999); Australasian Triage Scale -ATS ( Utilizado na Austrália, desde 
meados dos anos 70); Canadian Triage and Acuity Scale (CTAS) e Sistema de Triagem de Manchester - STM (foi criado pelo Manchester Triage Group e começou a ser utilizado nos serviços de emergência do Reino Unido a partir de 1996 e no Brasil em 2008), por esse motivo este sistema estar sendo utilizado neste artigo.

O STM, possui cinco categorias ou níveis. A cada categoria é atribuído um número, cor, nome e tempo-limite máximo aceitável até o primeiro atendimento com o médico. Os níveis são: nível 1: emergente, cor vermelho, atendimento imediato; nível 2: muito urgente, cor laranja, atendimento no máximo em 10 minutos; nível 3: urgente, cor amarelo, atendimento em até 60 minutos; nível 4: pouco urgente, cor verde, atendimento em até 120 minutos; nível 5: não urgente, cor azul, atendimento em até 240 minutos (Coutinho; Cecílio; Mota, 2018).

O objetivo do STM é identificar e separar rapidamente o doente em situação de risco iminente de morte e que precisa de atendimento imediato, dos que podem esperar mais tempo e com segurança o atendimento médico. A triagem é realizado pelo profissional enfermeiro, onde este é o responsável pela entrevista, exame físico e verificação dos sinais vitais, e a partir daí realizar a correta classificação de risco desse paciente. Este também deve avaliar a dor, como um importante indicador para medir a urgência do atendimento. Realizando reavaliação constante desse paciente, e enfim, defini a complexidade do caso, bem como o fluxo interno e a área mais adequada para atendimento do paciente. (Coutinho; Cecílio; Mota, 2018). Um dos problemas enfrentados pelo enfermeiro para esse atendimento, muitas vezes é a superlotação, que dificulta esse atendimento.

Considerando a importância da implantação e realização correta do Sistema de classificação de risco, nos serviços de atendimentos prestados a pacientes das mais diversas patologias, enfermidades, ou condições de saúde. E assim, possibilitando detectar precocemente o nível de gravidade e/ou risco de cada paciente. Assim, cada paciente ao buscar atendimento, seja priorizado sua necessidade e dessa maneira oferecido uma assistência de forma individualizada, evitando assim danos futuros, decorrentes a uma assistência tardia quando o paciente precisa ser atendido urgente.

Logo, esse trabalho visa ressaltar a importância do protocolo de Manchester de classificação de risco, identificando o conhecimento dos usuários acerca desse sistema, visando evitar danos à saúde dos pacientes decorrentes da insatisfação ocasionada muitas vezes pela classificação dos usuários recebida durante a triagem, em uma Unidade de Pronto Atendimento UPA localizada no município de Imperatriz - MA, no bairro São José.

Os usuários são uma peça-chave nos atendimentos das instituições de saúde, onde estes devem estar devidamente orientados, sobre a CR e assim, compreender o porquê de ter recebido determinada CR durante a triagem. Importante ressaltar que atualmente a maioria da população não tem conhecimento da importância da CR e dessa forma muitas vezes o desfecho dos atendimentos em saúde nem sempre são bem-sucedidos.

Visando contribuir, com a melhoria da assistência prestada, priorizando de forma correta os pacientes que necessitam de atendimentos urgentes. Nesse sentido, esse trabalho é voltado a pacientes que procuram atendimento nas Unidades de Saúde. Visando beneficiar também, a comunidade científica, fornecendo um melhor conhecimento a respeito dos usuários que são atendidos nos setores de urgência e emergência, no tocante a classificação de risco.

\section{Metodologia}

Como procedimento metodológico, a pesquisa será do tipo descritiva exploratória, com abordagem quantitativa e quanto aos procedimentos técnicos será do tipo pesquisa survey, para tanto utilizou- se como autor para o embasamento metodológico os dados de (Koche, 2011), uma vez que para se realizar uma pesquisa exploratória cria-se certa aproximação com o objeto de investigação. Menciona ainda, que numa pesquisa exploratória, são envolvidos levantamentos de bibliografias, entrevistas com componentes diretos do assunto, assim bem como, a análise e compreensão do problema abordado (Gil, 2011).

A pesquisa foi realizada através de um questionário com questões fechadas, sendo aplicado a pessoas adultas acima de 18 anos, que estavam aguardando atendimento na Unidade de Pronto Atendimento (UPA), localizada no bairro São José em 
Imperatriz - MA. O questionário foi aplicado no mês de agosto, setembro e outubro de 2021, no total foram entrevistadas 100 pessoas.

Os critérios de inclusão foram: Adultos acima de 18 anos de ambos os sexos; que na ocasião estavam aguardando atendimento na UPA São José e que concordaram em participar da pesquisa. Os critérios de exclusão foram: crianças e menores de 18 anos, que no momento estavam na UPA por outros motivos que não estejam em busca de atendimento médico e os que não concordarem em participar da pesquisa e não aceitarem assinar o termo de consentimento livre e esclarecido.

Como toda pesquisa com seres humanos, esta pesquisa foi realizada após aprovação do Comitê de Ética em Pesquisa (CEP), e respeitadas às normas de pesquisa envolvendo seres humanos, Resolução n 466/12 do Conselho Nacional de Saúde, autorização de $\mathrm{n}^{\mathrm{o}}$ 4.840.016 de 11 de julho de 2021.

\section{Resultados e Discussão}

Os artigos científicos selecionados para esta pesquisa, foram publicados em 2013 á em 2020, considerados produções científicas atuais sobre a classificação de risco. Os artigos foram lidos e organizados nas fundamentações da literatura o que deu respalda para a fundamentação da pesquisa de campo. No ato da pesquisa, foi assegurado aos entrevistados a cordialidade necessária, explicou-se sobre o motivo da pesquisa/orientação/informação, e a transparência do motivo da pesquisa, e após ter sido lido o TCLE e assinado pelo usuário a pesquisa foi realizada.

Os dados foram organizados em gráficos tipo pizza, onde no gráfico 1, temos o gênero dos usuários entrevistados, onde dos 100 usuário participante. 59\% eram do sexo feminino e 41\% do sexo masculino. Dos 100 participantes todos eram adultos de ambos os sexos, acima de 18 anos; todos estavam aguardando atendimento na UPA São José, em Imperatriz - MA e concordarem em participar da pesquisa. E dessa forma, 21\% dos participantes tinham idade 18 a 25 anos. $6 \%$ idade de 26 a 30 anos; $17 \%$ tinham idade de 31 a 36 anos. E 14\% tinham idade de 36 a 40 anos; 17\% dos participantes tinha de 40 anos a 50 ; $15 \%$ tinham de 51 a 60 anos; $9 \%$ acima de 60 anos e 14\% acima de $80 \%$ (Gráfico 1).

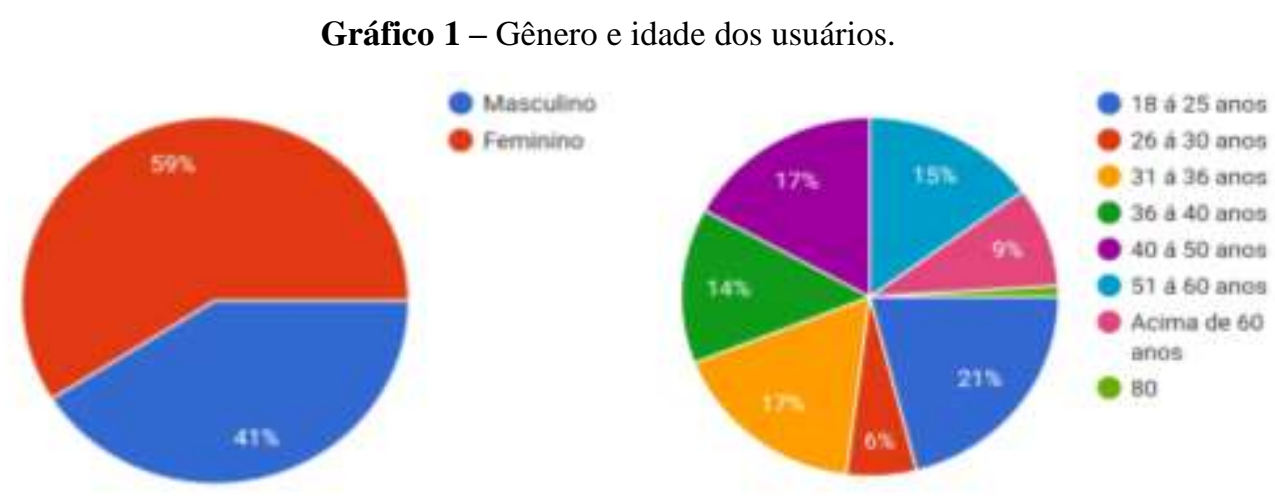

Fonte: Autores.

Quanto a escolaridade desses participantes variou entre: 5\% analfabetos; 25\% com Ensino Fundamental incompleto; 10\% Ensino Fundamental completo; 6\% Ensino Médio incompleto; 50\% Ensino Médio e 5\% Ensino Superior e 5\% pósgraduação (Gráfico 2). 
Gráfico 2 - Nível de escolaridade dos usuários.

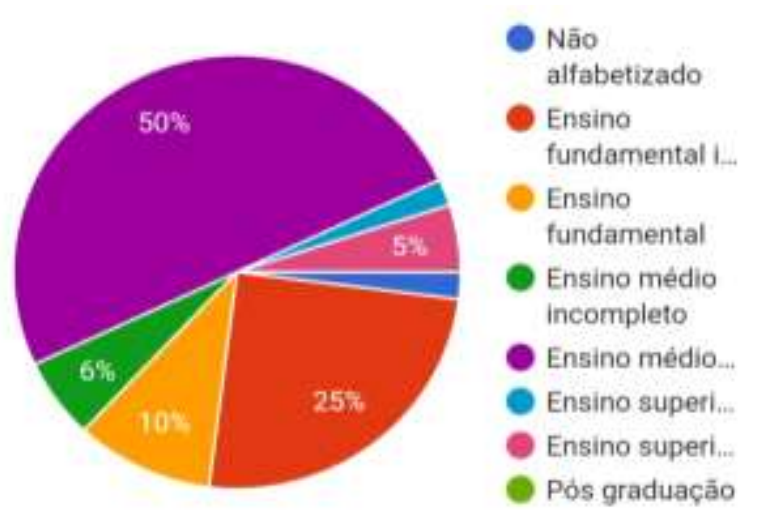

Fonte: Autores.

A Constituição Federal de 1988, art. 205, garante que "a Educação é um direito de todos, visando o pleno desenvolvimento da pessoa [...]" (Brasil, 1988). Porém, o que se vê é um país com cerca de $9 \%$ de analfabetos acima de 10 anos, o que equivale a 18 milhões de brasileiros (Ibge, 2018). De acordo com, de Oliveira et al (2019) a pobreza está fortemente correlacionada ao baixo nível de escolaridade. A redução da pobreza e da desigualdade social passa pela democratização do ensino de qualidade. E a pobreza é uma das questões de sobrecarregar o sistema de saúde, uma vez que pessoas consideradas de renda alta tende a procurar o sistema particular de saúde. Dos 100 participantes que responderam ao questionário a respeito se conheciam a importância da Triagem para o atendimento, $68 \%$ responderam que sim, e $32 \%$ que não sabiam da importância da Triagem para o atendimento (Gráfico 3).

Gráfico 3 - Conhecimento do usuário sobre a importância da Classificação de Risco.

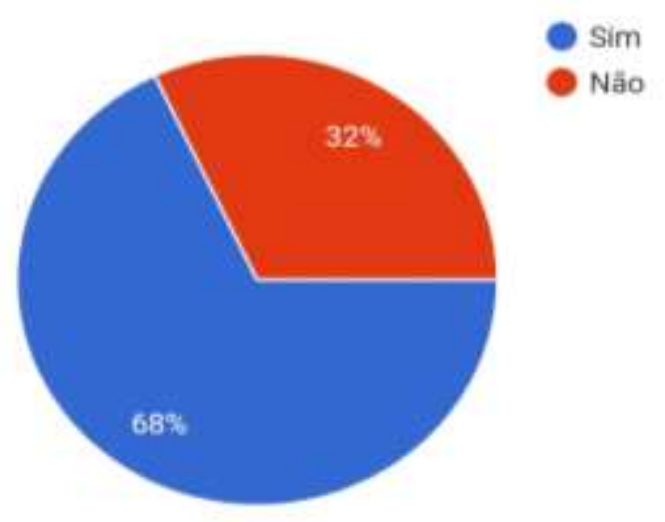

Fonte: Autores.

De acordo com o Brasil (2002), a triagem classificatória deverá ser realizada por meio de protocolos préestabelecidos, por profissionais de saúde de nível superior, com treinamento específico; sendo proibida a dispensa de pacientes antes que estes recebam atendimento médico.

“A grande vantagem desta Triagem é separar os casos verdadeiramente urgentes dos não urgentes e garantir o atendimento prioritário dos casos mais graves", explica a enfermeira. Ela ainda informa que os pacientes deixam de ser atendidos pela ordem de chegada ao setor de urgência e passam a ser em função da gravidade da situação. "É um grande passo para a sistematização da assistência. O fato de os doentes estarem ordenados por prioridades é vantajoso para os profissionais, 
que passam a ter uma imagem clara do número de doentes que se encontram no setor e da sua gravidade, permitindo gerir as tarefas a atuar de forma mais correta e responsável” (Coren, 2009).

Quanto a resposta se sabia para que serve a classificação de risco, 52\% responderam que sim enquanto $48 \%$ afirmaram não saberem para que serve a classificação de risco (Gráfico 4).

Gráfico 4 - Conhecimento do usuário sobre o protocolo de classificação de Risco.

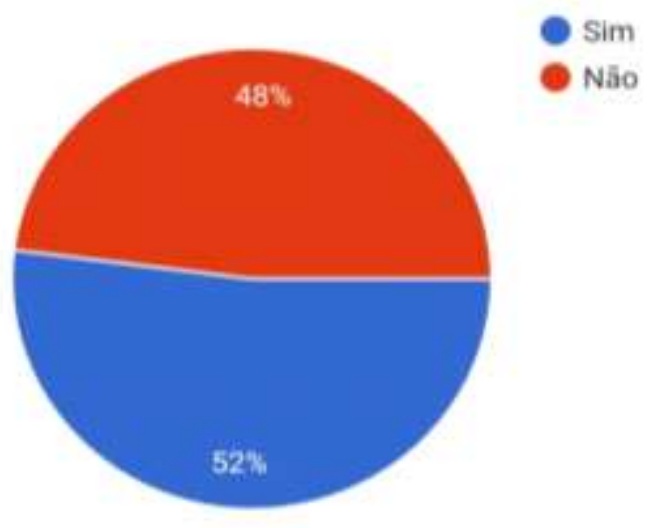

Fonte: Autores.

Em 2004, foi instituída a Política Nacional de Classificação de Risco (Humaniza SUS), tendo em vista que os atendimentos por ordem de chaga já não eram mais eficazes para o atendimento de parcelas de população crescente, principalmente nas Unidades de Pronto Atendimento, evidenciando a escassez da assistência primária e de recursos humanos (Brasil, 2010).

Segundo Brasil (2010), o acolhimento com avaliação eclassificação de risco é um esforço de melhorar e concretizar os princípios determinados pelo SUS, funcionando como um instrumento de humanização da saúde, pois busca um trabalho coletivo e cooperativo, uma postura ética, respeitando as diferenças dos sujeitos. Esse acolhimento, portanto, é uma das ações do Programa Nacional de Política de Humanização (PNH), onde neste programa prevalece o atendimento humanizado e de qualidade respeitando o sofrimento de todos aqueles que procuram o serviço de emergência.

Onde se perguntou, se os participantes sabem o significado da cor que recebe quando feito a classificação de risco, $54 \%$ deles responderam que sim e $46 \%$ responderam que não sabem o significado da cor (Gráfico 5).

Gráfico 5. Conhecimento dos usuários sobre o significado das cores.

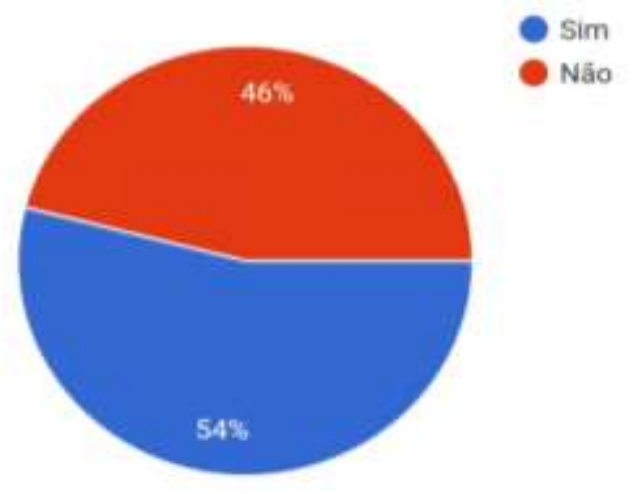

Fonte: Autores. 
No atendimento pelo ACCR (Acolhimento com Classificação e Avaliação de Risco), após o usuário ser acolhido no serviço, utiliza-se o ordenamento estratificado na forma de, no mínimo, quatro cores para classificar os agravos: vermelho (emergência); amarelo (urgência); verde (menor urgência); e azul (não urgência), representando, respectivamente, a escala do maior ao menor risco, segundo critérios clínicos estabelecidos por protocolos institucionalizados (Da Luz, 2019).

A classificação de risco trabalha com cores que identificam a real necessidade do indivíduo, tais elas são: Vermelha Emergência: atendimento imediato; Laranja - Muito urgente: espera de até 10 minutos; Amarelo - Urgente: espera de até 1 hora; Verde - Pouco urgente: espera de até 2 horas; Azul - Não urgente: espera de até 4 horas (Roncalli et al 2017).

Dos 100 participantes que responderam ao questionário, na questão se achavam importante a classificação de risco, 91\% responderam que sim e 9\% responderam que não achavam importante a classificação de risco (Gráfico 6).

Gráfico 6. Conhecimento dos usuários sobre a importância do protocolo de classificação de risco.

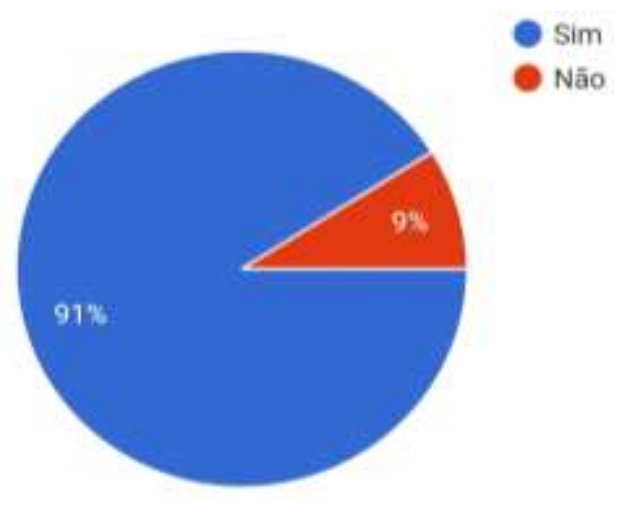

Fonte: Autores.

Segundo Sacoman et al, (2019), a metodologia de classificação de risco apresenta vantagens por controlar a demanda, otimizar o atendimento as urgências e emergências e diminuir a sobrecarga ocupacional da equipe de saúde e fortalecer o vínculo usuário - trabalhador.

Para Chabudè (2019) os critérios de classificação do risco clínico dos pacientes, situados em ambos os protocolos contêm os níveis de prioridade e cor atribuída, o tempo de espera para o atendimento médico, a reavaliação de enfermagem e o método de avaliação do motivo principal, estratificando o risco em cinco níveis distintos e que têm sido definidos por cores para facilitar a visualização.

De acordo com Paz, (2019), na percepção de usuários a classificação de risco se torna excludente, pois o profissional de saúde irá ter que escolher quem será atendido, um dado a ser questionado em relação a aceitação da sociedade a respeito do método de classificação de risco. Que na percepção do usuário, a classificação de risco é rápida, pois atende quem está mais grave e quem está menos grave pode aguardar.

Quanto ao atendimento na UPA, foi perguntado se todas as vezes receberam uma cor para o atendimento, $76 \%$ responderam afirmativamente, enquanto $24 \%$ disseram que não receberam (Gráfico 7). 
Gráfico 7. Sobre a eficácia na aplicabilidade do protocolo de classificação de risco.

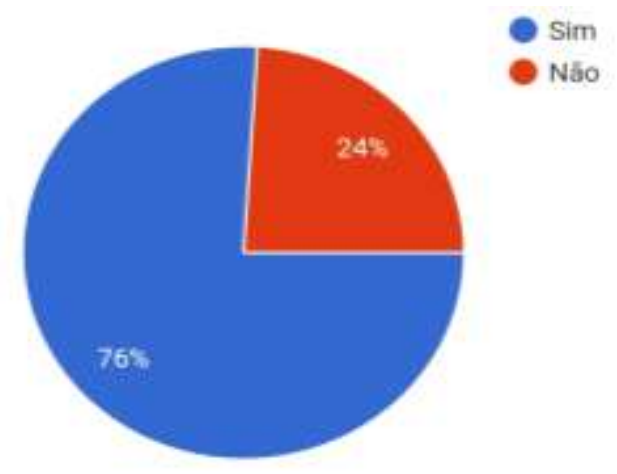

Fonte: Autores.

Ainda, os serviços emergenciais podem adotar sistemas com outras categorias para o atendimento, ao exemplo do protocolo de Manchester, que é muito utilizado globalmente e acrescenta a cor laranja aos pacientes classificados como "muito urgentes”, ou seja, um intermédio entre as cores vermelha e amarela antes citadas (Araujo, 2017).

\section{Conclusão}

No total foram entregues 100 questionários fechados para 100 sujeitos, sendo 59 mulheres e 41 homens. A idade variou entre 18 e 80 anos. Quanto a escolaridade desses participantes variou entre: 5\% analfabetos; 25\% com Ensino Fundamental incompleto; 10\% Ensino Fundamental completo; 6\% Ensino Médio incompleto; 50\% Ensino Médio e 5\% Ensino Superior e $5 \%$ pós-graduação.

Dos 100 participantes que responderam ao questionário a respeito se conheciam a importância da Triagem para o atendimento, $68 \%$ responderam que sim, e 32\% que não sabiam da importância da Triagem para o atendimento. No que se refere a classificação de risco, quanto a resposta quanto ao conhecimento sobre a importância do protocolo de Manchester de classificação de risco, $52 \%$ responderam que sim enquanto $48 \%$ afirmaram não saberem para que serve a classificação de risco.

Assim, a partir dos discursos elaborados pelos sujeitos, foi possível perceber a percepção deles quanto a importância da classificação de risco ao atendimento na UPA São José conforme os gráficos acima mostram e conclui-se que a baixa escolaridade trás sim, mais desigualdade social e com isto o acarretamento da superlotação na saúde pública. Quanto a importância do protocolo de classificação de risco, percebe-se que devido ao grande número de pessoas nas filas a procura de atendimento de urgência, com a efetividade da aplicabilidade de classificação de risco os usuários são atendidos de acordo com a gravidade e não mais por ordem de chegada.

Nesse sentido, faz-se necessário ações, visando a capacitação constante dos profissionais enfermeiros, no sentido de perceber a importância desse protocolo para a efetividade dos serviços de saúde, uma vez que muitas vezes devido à alta demanda de serviços diários, muitas vezes o protocolo de Manchester deixa de ser aplicado de forma correta, acarretando classificação erradas e consequentemente impacto na qualidade dos serviços prestado. Nesse sentido, faz-se necessários mais estudo a respeito do assunto, no sentido de trazer a luz uma problemática existente e que impacta negativamente na qualidade da assistência prestada. 


\section{Referências}

Araújo, J. H. D. V., Chaves, G. M. M., Barbosa, J. V., \& Nunes, C. M. P. (2017). Compreensão dos usuários do SUS sobre a classificação de risco na rede de urgência e emergência por meio de um programa de educação tutorial.

Barbosa L. A. S., Sauthler, M., Paes, G. O., \& Teixeira, E. R. (2019). Acolhimento com classificação de risco: relação de justiça com o usuário. Revista Brasileira de Enfermagem, 72(6).

Brasil. (1988). Presidência da República. Constituição da República Federativa do Brasil de $1988 . \quad$ Brasília, DF, Senado. https://www2.senado.leg.br/bdsf/bitstream/handle/id/518231/CF88_Livro_EC91_2016.pdf

Brasil. (2002). Ministério da Saúde. Gabinete do Ministro. Portaria n $\mathrm{n}^{\mathrm{o}} 2.048$, de 05 de novembro de 2002. https://bvsms.saude.gov.br/bvs/saudelegis/gm/2002/prt2048_05_11_2002.html

Brasil. (2010). Ministério da Saúde. Secretaria de Atenção à Saúde. Núcleo Técnico da Política Nacional de Humanização. Acolhimento nas práticas de produção de saúde / Ministério da Saúde, Secretaria de Atenção à Saúde, Núcleo Técnico da Política Nacional de Humanização. - (2ª ed.) 5. reimp. - Brasília: Editora do Ministério da Saúde, 2010. 44 p.: il. color. - (Série B. Textos Básicos de Saúde

Chabudé, T. G., César, G. C., \& Santana, C. J. (2019). Acolhimento e Classificação de Risco em Unidade de Urgência: Relato de Experiência da Implantação do Sistema de Triagem de Manchester. Ensaios e Ciência C Biológicas Agrárias e da Saúde, 23(2), 121-125.

Coelho, B. (2019). Tipos de pesquisa: abordagem, natureza, objetivos e procedimentos. https://blog.mettzer.com/tipos-de-pesquisa/.

COREN (2009). Conselho Regional de Enfermagem. São Paulo, Brasil. https: manual-anotacoes-de-enfermagem-coren-sp.pdf (portaldaenfermagem.com.br)

Coutinho, A. A. P., Cecílio, L. C. D. O., \& Mota, J. A. C. (2012). Classificação de risco em serviços de emergência: uma discussão da literatura sobre o Sistema de Triagem de Manchester. Rev Med Minas Gerais, 22(2), 188-98.

de Oliveira, B. R., \& de Lacerda Peixoto, M. D. C. (2019). Educação, Pobreza e Programas de Transferência de Renda: A Implementação do Programa Oportunidades no México. Education Policy Analysis Archives, 27.

da Luz, S. (2019). Acolhimento humanizado ao paciente atendido no Pronto Atendimento de um Hospital Geral da rede privada de Belo Horizonte, Minas Gerais.

dos Santos, S., Gomes, D. C., dos Santos, M. A. A. C., Bezerra, D. G., \& dos Reis, R. P. (2020). A atuação do enfermeiro na classificação de risco de pacientes em unidade de emergência: um enfoque no protocolo de Manchester. Revista Eletrônica da Estácio Recife.

Gil, A. C. (2011). Metodologia do ensino superior. (4ª ed.) São Paulo: Atlas.

Koche, J. C. (2011). Fundamentos de metodologia científica. Petrópolis: Vozes. http://www.brunovivas.com/wpcontent/uploads/sites/10/2018/07/K\%C3\%B6che-Jos\%C3\%A9-Carlos0D0AFundamentos-de-metodologia-cient\%C3\%ADfica-_teoriada0D0Aci $\%$ C3\%AAncia-e-inicia\%C3\%A7\%C3\%A3o-\%C3\%A0-

pesquisa.pdfhttps://repositorio.ufsm.br/bitstream/handle/1/15824/Lic_Computacao_Metodologia-Pesquisa-Cientifica.pdf?sequence=1

Melo, M. do C. B. de. (2011). Urgência e Emergência na Atenção Primária à Saúde / Maria do Carmo Barros de Melo e Nara Lúcia Carvalho da Silva. -- Belo Horizonte: Nescon/UFMG. 132p.: il., 22x27cm.

Oliveira, J. L. C. D., Gatti, A. P., Barreto, M. D. S., Belucci, J, J. A., Góes, H. L. D. F., \& Matsuda, L. M. (2017). Acolhimento com classificação de risco: percepções de usuários de uma unidade de pronto atendimento. Texto \& Contexto-Enfermagem, 26(1).

Paz, D. S. (2019). Percepção do usuário quanto a atuação do enfermeiro na sala de classificação de risco em um serviço de urgência e emergência.

Rocha. Sônia. (2013). Pobreza no Brasil: afinal, de que se trata? Rio de Janeiro: Editora FGV.

Roncalli, A. A. de O. D. N., Silva, I. C. M., Brito, R. F., \& DA Fonseca, V. S. M. (2017). Protocolo de Manchester e população usuária na classificação de risco: visão do enfermeiro. Revista Baiana de Enfermagem31,(2).

Sacoman, T. M., Beltrammi, D. G. M., Andrezza, R., Cecílio, L. C. D. O., \& Reis, A. A. C. D. (2019). Implantação do Sistema de Classificação de Risco Manchester em uma rede municipal de urgência. Saúde em Debate, 43, 354-367.

Spanuolo, R. S., Silva, M. N. L., Meneguin, S., Basseto, J. G. B., \& Fernandes, V. C. (2017). Percepção dos usuários sobre a triagem com classificação de risco em um serviço de urgência de cabo verde. Revista Brasileira em Promoção da Saúde, 30(2), $249-254$.

Souza, C. C. D., Toledo, A. D., Tadeu, L. F. R., \& Chianca, T. C. M. (2011). Classificação de risco em pronto-socorro: concordância entre um protocolo institucional brasileiro e Manchester. Revista Latino-Americana de Enfermagem, 19, 26-33.

Mackway-Jones, K., Marsden, J., \& Windle, J. Emergency Triage. Manchester Triage Group. (2a ed.) Oxford: Blackwell; 2006. 178p. 